\title{
The Relationship between Type D Personality and Heart Rate Variability in Community Mental Health Center Users
}

\author{
Noeul Kang ${ }^{1}$, Jeung-Suk Lim², Taik-Gun Hwang ${ }^{3}$, Sook-Haeng Joe ${ }^{4,5}$, and Moon-Soo Lee ${ }^{2,4,5} \bowtie$ \\ ${ }^{1}$ Korea University School of Medicine, Seoul, Republic of Korea \\ ${ }^{2}$ Guro Community Mental Health Center, Seoul, Republic of Korea \\ ${ }^{3}$ Guro Public Health Center, Seoul, Republic of Korea \\ ${ }^{4}$ Department of Psychiatry, Korea University Medical Center, Seoul, Republic of Korea \\ ${ }^{5}$ Korea University Research Institute of Mental Health, Seoul, Republic of Korea
}

\begin{abstract}
Objective Type D personality can be regarded as a promising cardiovascular risk marker that has been repeatedly linked to relevant indicators of mental health, quality of life, morbidity, and mortality in cardiac patients. Heart rate variability (HRV) is a non-invasive technology that can provide information regarding a patient's sympathetic/parasympathetic balance and the control mechanisms of the autonomic systems in the cardiovascular system. As both type D personality and HRV are parameters related to the cardiovascular system, we assumed a relationship between type D personality and HRV. This study set out to identify the relationship between type D and $\mathrm{HRV}$ and the differences in HRV variables between type $\mathrm{D}$ and non-type $\mathrm{D}$ personalities.
\end{abstract}

Methods Patients who visited Guro Community Mental Health Center from January 2011 to December 2012 were surveyed. They were evaluated using both the Korean version of the Type D Personality-14 for type D personality and HRV. During the survey, those who reported major cardiovascular disease that can affect heart rate variability were excluded from the study.

Results Our analysis included 559 participants, 249 of whom were classified as type D personality. No significant differences were found in the HRV variables between the type D group and the non-type D group. There were also no clinically meaningful correlations between HRV variables and type D total/subscale scores when controlled for patient age.

Conclusion A relationship between HRV and type D personality was not identified using short-term HRV measurements in non-clinical patients with no definitive cardiovascular disease. Further studies using long-term HRV measurements in patients with cardiovascular disease are necessary to conclude an association between HRV and type D personality.

Psychiatry Investig 2015;12(2):197-203

Key Words Type D personality, Heart rate variability, Cardiovascular disease, Autonomic instability, Short-term measurement.

\section{INTRODUCTION}

Heart disease is the leading cause of death for both men and women in the United States. Coronary heart disease is the most common type of heart disease. ${ }^{1}$ In addition, the cost of cardiac care continues to increase as patients with cardiovascular disease are living longer. ${ }^{2}$ As a result, cardiovascular diseases (CVD), which include heart attack, stroke, heart failure, and other conditions, are an important issue in public

Received: January 17, 2014 Revised: June 12, 2014

Accepted: July 7, 2014 Available online: January 20, 2015

$\bowtie$ Correspondence: Moon-Soo Lee, MD, PhD

Department of Psychiatry, Korea University Medical Center, 148 Gurodongro, Guro-gu, Seoul 152-703, Republic of Korea

Tel: +82-2-2626-3163, Fax: +82-2-852-1937, E-mail: npboard@gmail.com

(a) This is an Open Access article distributed under the terms of the Creative Commons Attribution Non-Commercial License (http://creativecommons.org/licenses/bync/3.0) which permits unrestricted non-commercial use, distribution, and reproduction in any medium, provided the original work is properly cited. health practice.

Several studies have shown that cardiovascular diseases are caused by psychological as well as physiological factors, such as psychosocial conditions and behavioral mechanisms; these risk factors contribute significantly to the pathogenesis of CVD. ${ }^{3,4}$ In general, personality can be defined as the dynamic individual makeup of psychobiological systems that modulates his or her unique adaptation to a changing environment. ${ }^{5}$ Cardiovascular research has reported extensively on the role of personality in ischemic heart disease. ${ }^{6}$ A study of coping styles in patients with coronary heart disease proposed a new personality construct, known as type D or "distressed" personality. ${ }^{7}$

Theories of personality and empirical evidence have drawn attention to type $\mathrm{D}$ personality as a potential risk factor for CVD instead of type A personality. ${ }^{7}$ This discrete, homogeneous, distressed personality type was originally recognized 
in European patients with ischemic heart disease. Type D personality is characterized by normal and stable personality traits, negative affectivity, and social inhibition. Negative affectivity is the tendency to experience negative emotions, while social inhibition refers to the inhibited expression of emotions. ${ }^{8}$ Type D personality is also associated with an increased risk for impaired quality of life, morbidity, and mortality in conjunction with various CVDs. ${ }^{9}$ It has been suggested as a prognostic predictor and a determinant of clinical outcome and health status, even after therapeutic interventions. ${ }^{10}$ The biological mechanisms underlying this association are poorly understood; however, the negative health impact could be caused by 1) an increased risk for mental disorders like depression and anxiety, which have also been identified as risk factors for $\mathrm{CHD}, 2$ ) poor treatment adherence and an unhealthy lifestyle, and 3) underlying biological mechanisms, such as inflammation or autonomic imbalance. ${ }^{11}$

Heart rate variability (HRV) refers to the fact that heart rate is not always stable but constantly fluctuates around a mean value. This fluctuation is influenced by the autonomic nervous system, which regulates heart rate through the sympathetic and parasympathetic nervous systems; ${ }^{12}$ these cyclic changes in sinus rate are termed heart rate variability. ${ }^{13} \mathrm{HRV}$ has been used as an indicator of autonomic nervous system function in many settings. ${ }^{14}$ In stable conditions, heart rate intervals are not regular and constantly change, even at microscopic levels; this physiological mechanism allows the human body to maintain homeostasis. A healthy person maintains a high HRV and is therefore ready to respond to various stimulations. When the autonomic nervous system fails, the HRV decreases, producing an increased susceptibility to stress. Low HRV has been associated with excessive cardiac sympathetic modulation, inadequate parasympathetic modulation, or both. In addition, psychological stress influences the cardiovascular system via the autonomic nervous system. ${ }^{15}$ Study of HRV is a non-invasive monitoring method that offers information about the activities of the autonomic nerves that innervate the heart. This method is used to evaluate functions of the autonomic nervous system by analyzing its influences on the cardiovascular system. A relationship between impaired autonomic function measured by HRV and an increased risk of mortality was shown in patients who were already at risk because they had diabetes. ${ }^{16}$

However, the associations of these measures of autonomic function (parameters of HRV) with type D personality have never been compared in a single study population. Therefore, we studied the associations of several parameters of HRV with type $\mathrm{D}$ personality in a community sample. The primary study aim was to evaluate the relationship between type D personality and HRV in community samples.

\section{METHODS}

\section{Participants and procedures}

We investigated community members enrolled in the Guro-gu Mental Health Promotion Program from January 2011 to December 2012. This program was a population-based, integrated community mental health service provided by Guro Community Mental Health Center in the southwestern region of Seoul, Korea.

Community members who visited Guro Community Mental Health Center were evaluated for type D personality and HRV and responded to survey forms. Those who reported a history of major cardiovascular disease, such as heart attack, stroke, heart failure, or coronary heart disease, were excluded from this study, as our focus was on the relationship between HRV and type D personality without any other confounding factors.

\section{Measures}

The Korean Version of the Type D Personality-14 (DS14)

Type D personality was assessed with the 14-item DS14, which measures the two subscales of negative affectivity (NA) and social inhibition (SI). Each item is rated according to a five-point Likert-type scale ranging from 0 to 4 . Participants are classified as having type $\mathrm{D}$ personality if they score high on both the NA and the SI using cutoff of 10 on both scales (i.e., NA $\geq 10$ and $\mathrm{SI} \geq 10$ ). The Cronbach's alpha of the original DS14 was 0.88 for the NA and 0.86 for the SI, respectively, and test-retest reliability was 0.72 and $0.82 .{ }^{8}$ The Korean translation of the DS-14 was validated in a previous communitybased study. ${ }^{17}$

\section{Evaluation of heart rate variability}

Data collection and HRV analysis were performed using HRV analytic equipment SA-2000E (Medicore Company, Korea). To control diurnal variation, HRV was measured between 8 a.m. and 12 p.m. in a quiet environment at room temperature. The recordings were made over a period of $5 \mathrm{~min}$. Each participant was seated in a chair, and electrodes were placed on the wrists and left foot. The participant was instructed to breathe at a normal rate during the HRV measurement. Before the measurement, participants rested for at least $10 \mathrm{~min}$, breathing spontaneously without talking.

The parameters measured in the evaluation of HRV were grouped into two categories: time domain and frequency domain. For the time domain, the standard deviation of the normal-to-normal interval (SDNN) was used to estimate longterm components of the HRV, and the square root of the mean squared differences of successive normal-to-normal intervals 
(RMSSD) was calculated using statistical time domain measurements. The following HRV parameters were also measured by frequency domain spectral analysis: total power (TP), very low frequency (VLF), low frequency (LF), and high frequency (HF). The LF/HF ratio was also calculated because it is considered to reflect a simultaneous effect on both sympathetic and vagal modulation. ${ }^{18}$

\section{Statistical analysis}

Participants' demographic features were analyzed using descriptive statistics. Group comparisons between type D personality and non-type D personality for baseline characteristics were made using independent t-tests for continuous variables. The correlations between type $\mathrm{D}$ total/subscale scores and HRV indices were investigated using Pearson correlation coefficients in each participant group (total, non-type D participants, and type D participants) after controlling for age.

All analyses were performed using the Statistical Package for the Social Sciences (SPSS) version 21.0 for Windows. A pvalue $<0.05$ was considered statistically significant.

\section{Ethics statement}

The present study protocol was approved by the Institutional Review Board of Korea University Guro Hospital in Seoul, Korea. All of the participants provided written informed consent before the study began.

\section{RESULTS}

This study initially had 562 participants, but three were ex- cluded from the analysis due to missing data. As a result, 559 participants (204 males, 355 females) were included in the final analyses. The mean age of the participants was $49.97 \pm$ 17.20 years (range: $18-92$ years); males and females had mean ages of $52.37 \pm 17.47$ and $48.60 \pm 16.91$ years, respectively. There were 249 participants with type D personality and 310 who were identified as having non-type D personality. No significant age difference was found between the type $\mathrm{D}$ and the non-type D personality groups. The characteristics of each group and the age and sex distributions of participants with type $\mathrm{D}$ personality are summarized in Table 1.

The mean SDNN and RMSSD values for all participants

Table 1. Demographic characteristics of participants

\begin{tabular}{cc}
\hline Total & 559 \\
Age & $49.97 \pm 17.20$ \\
Sex (\%) & \\
Male & $204(36.5)$ \\
Female & $355(63.5)$ \\
Type D (\%) & $249(44.5)$ \\
Age & $49.65 \pm 17.42$ \\
Sex (\%) & \\
Male & $89(35.7)$ \\
Female & $160(64.3)$ \\
Non-type D (\%) & $310(55.5)$ \\
Age & $50.24 \pm 17.03$ \\
Sex (\%) & \\
Male & $115(37.1)$ \\
Female & $195(62.9)$ \\
\hline
\end{tabular}

Table 2. Comparison of type D subscale scores and HRV parameters between type D and non-type D personality participants

\begin{tabular}{|c|c|c|c|c|}
\hline & Total & Type D & Non-type D & $\mathrm{p}$-value \\
\hline Type D total score & $20.40 \pm 12.93$ & $31.58 \pm 8.56$ & $11.43 \pm 7.86$ & 0.001 \\
\hline \multicolumn{5}{|c|}{ Type D subscale score } \\
\hline NA & $9.16 \pm 6.96$ & $15.76 \pm 4.30$ & $3.86 \pm 3.06$ & 0.001 \\
\hline SI & $11.24 \pm 7.00$ & $15.82 \pm 5.59$ & $7.56 \pm 5.75$ & 0.001 \\
\hline \multicolumn{5}{|l|}{ HRV parameters } \\
\hline Mean heart rate & $77.80 \pm 28.01$ & $77.34 \pm 26.32$ & $78.17 \pm 29.33$ & NS \\
\hline SDNN (ms) & $33.15 \pm 18.29$ & $33.67 \pm 20.75$ & $32.74 \pm 16.06$ & NS \\
\hline RMSSD (ms) & $27.40 \pm 17.50$ & $27.36 \pm 18.23$ & $27.43 \pm 16.93$ & NS \\
\hline $\mathrm{TP}\left(\mathrm{ms}^{2}\right)$ & $978.96 \pm 1441.72$ & $1079.40 \pm 1819.48$ & $898.28 \pm 1040.13$ & NS \\
\hline $\operatorname{VLF}\left(\mathrm{ms}^{2}\right)$ & $460.60 \pm 1014.49$ & $560.59 \pm 1418.41$ & $380.28 \pm 478.85$ & NS \\
\hline $\mathrm{LF}\left(\mathrm{ms}^{2}\right)$ & $303.06 \pm 530.29$ & $294.53 \pm 424.18$ & $309.91 \pm 602.74$ & NS \\
\hline $\mathrm{HF}\left(\mathrm{ms}^{2}\right)$ & $215.30 \pm 292.27$ & $224.27 \pm 316.22$ & $208.09 \pm 271.82$ & NS \\
\hline $\mathrm{LF} / \mathrm{HF}$ & $2.29 \pm 4.66$ & $2.12 \pm 2.30$ & $2.42 \pm 5.92$ & NS \\
\hline
\end{tabular}

Numbers are presented as mean \pm standard deviation. NA: negative affectivity, SI: social inhibition, HRV: heart rate variability, SDNN: standard deviation of the normal to normal interval, RMSSD: square root of the mean squared differences of successive normal to normal intervals, TP: total power, VLF: very low frequency, LF: low frequency, HF: high frequency, LF/HF: ratio of LF to HF, NS: not significant 
Table 3. Partial correlation between heart rate variability parameters and type D subscale scores after controlling for age in each participant group

\begin{tabular}{|c|c|c|c|c|c|c|c|c|}
\hline & Mean heart rate & SDNN & RMSSD & $\mathrm{TP}$ & VLF & LF & $\mathrm{HF}$ & $\mathrm{LF} / \mathrm{HF}$ \\
\hline \multicolumn{9}{|l|}{ Total } \\
\hline Total score & NS & NS & NS & NS & NS & NS & NS & NS \\
\hline NA score & NS & NS & NS & NS & NS & NS & NS & NS \\
\hline SI score & NS & NS & NS & NS & NS & NS & NS & NS \\
\hline \multicolumn{9}{|c|}{ Non-type D personality participants } \\
\hline Total score & NS & NS & NS & $-0.112^{*}$ & $-0.144^{*}$ & NS & NS & NS \\
\hline NA score & NS & NS & NS & NS & $-0.118^{*}$ & NS & NS & $-0.135^{*}$ \\
\hline SI score & NS & NS & NS & NS & $-0.134^{*}$ & NS & NS & NS \\
\hline \multicolumn{9}{|c|}{ Type D personality participants } \\
\hline Total score & NS & NS & NS & NS & NS & NS & NS & NS \\
\hline NA score & NS & NS & NS & NS & NS & NS & NS & NS \\
\hline SI score & NS & NS & NS & NS & NS & NS & NS & NS \\
\hline
\end{tabular}

${ }^{*} \mathrm{p}<0.05$. NA: negative affectivity, SI: social inhibition, HRV: heart rate variability, SDNN: standard deviation of the normal to normal interval, RMSSD: square root of the mean squared differences of successive normal to normal intervals, TP: total power, VLF: very low frequency, LF: low frequency, HF: high frequency, LF/HF: ratio of LF to HF, NS: not significant

were $33.15 \pm 18.29$ and $27.40 \pm 17.50$, respectively, in the timedomain analysis, while the mean TP, VLF, LF, HF, and LF/HF ratios of all participants were 978.96 $\pm 1441.72,460.60 \pm$ $1014.49,303.06 \pm 530.29,215.30 \pm 292.27$, and $2.29 \pm 4.66$, respectively, in the frequency-domain spectral analysis. The comparison results of type $\mathrm{D}$ personality total/subscale scores and the 5-minute HRV resting measurements in each participant group are summarized in Table 2. There was no significant difference in the HRV variables between type $D$ personality and non-type D personality (Table 2).

Ages influence on the heart rate variability and heart rate variation is known to decline with age. ${ }^{19}$ The correlations between type D personality variables (SI, NA) and HRV parameters were assessed after controlling for age, which is a known factor that influences HRV (Table 3). The total type D score (sum of the NA and SI subscale scores) showed significant correlations with TP ( $\mathrm{r}=-0.112)$ and VLF ( $\mathrm{r}=-0.144)$ in Nontype $\mathrm{D}$ personality participants. The NA score in non-type $\mathrm{D}$ personality participants had a significant negative correlation with VLF measurement $(r=-0.118)$ and LF/HF ratio $(r=$ -0.135). The SI score in non-type D personality participants also showed a significant negative correlation with VLF measurement $(r=-0.134)$. However, all of the correlation coefficients were low, even though they were statistically significant.

We divided the participants into two groups-adult age group (19-44 years), and middle aged and aged group (older than 45 years) according to age spectrum. The correlation analysis between type D personality variables and HRV parameters were assessed again after controlling for age, separately (Table 4). All of the correlation coefficients were low again, even though some parameters were statistically significant [Total type D score with TP $(\mathrm{r}=-0.188)$ \& LF $(\mathrm{r}=-0.219)$, NA score with $\mathrm{LF} / \mathrm{HF}$ ratio $(\mathrm{r}=-0.208)$, and SI score with $\mathrm{TP}(\mathrm{r}=-0.192)$ \& LF ( $r=-0.211)$ in Non-type D personality participants of adult age group; Total type $\mathrm{D}$ score with mean heart rate $(\mathrm{r}=$ $0.144)$ \& VLF ( $r=-0.156)$, NA score with mean heart rate $(r=$ $0.162)$, and SI score with VLF ( $r=-0.149)$ in Non-type D personality participants of middle aged and aged group].

\section{DISCUSSION}

People with type D personality have been reported to use more passive and maladaptive avoidance coping strategies, which are associated with higher levels of perceived stress and burnout symptoms. ${ }^{20}$ Systematic reviews and meta-analyses of type D studies have shown an association with poor cardiovascular outcomes. ${ }^{20,21}$

The average age of the participants was close to 50 years, and 249 of 559 participants, about $44.3 \%$ of the total study population, presented with type D personality. An assessment of the DS14 in the Korean population was reported in a previous study published by Korean researchers, where the prevalence of the type D construct was $27.8 \%$ in the general population (total number $=954$, mean age $=43.3 \pm 12.8$ years) ${ }^{17}$ As these two studies are performed in different clinical settings, it is not possible to make a direct comparison. However, the prevalence of type $\mathrm{D}$ personality tended to be higher in the present study population than it was in that of the previous one. We think that this difference might be due to the epidemiological characteristics of the community mental health center patients who participated in the study. Their mean age was relatively higher than that in the previous study. Clients 
Table 4. Partial correlation between heart rate variability parameters and type D subscale scores after controlling for age in each participant group divided by age (adult group/ middle aged and aged group)

\begin{tabular}{|c|c|c|c|c|c|c|c|c|}
\hline & Mean heart rate & SDNN & RMSSD & $\mathrm{TP}$ & VLF & $\mathrm{LF}$ & $\mathrm{HF}$ & $\mathrm{LF} / \mathrm{HF}$ \\
\hline \multicolumn{9}{|l|}{ Adult group } \\
\hline \multicolumn{9}{|l|}{ Total $(\mathrm{N}=216)$} \\
\hline Total score & NS & NS & NS & NS & NS & NS & NS & NS \\
\hline NA score & NS & NS & NS & NS & NS & NS & NS & NS \\
\hline SI score & NS & NS & NS & NS & NS & NS & NS & NS \\
\hline \multicolumn{9}{|c|}{ Non-type D personality participants $(\mathrm{N}=116)$} \\
\hline Total score & NS & NS & NS & $-0.188^{*}$ & NS & $-0.219 *$ & NS & NS \\
\hline NA score & NS & NS & NS & NS & NS & NS & NS & $-0.208^{*}$ \\
\hline SI score & NS & NS & NS & $-0.192^{*}$ & NS & $-0.211^{*}$ & NS & NS \\
\hline \multicolumn{9}{|c|}{ Type D personality participants $(\mathrm{N}=100)$} \\
\hline Total score & NS & NS & NS & NS & NS & NS & NS & NS \\
\hline NA score & NS & NS & NS & NS & NS & NS & NS & NS \\
\hline SI score & NS & NS & NS & NS & NS & NS & NS & NS \\
\hline \multicolumn{9}{|c|}{ Middle aged and aged group } \\
\hline \multicolumn{9}{|l|}{ Total $(\mathrm{N}=343)$} \\
\hline Total score & NS & NS & NS & NS & NS & NS & NS & NS \\
\hline NA score & NS & NS & NS & NS & NS & NS & NS & NS \\
\hline SI score & NS & NS & NS & NS & NS & NS & NS & NS \\
\hline \multicolumn{9}{|c|}{ Non-type D personality participants $(\mathrm{N}=194)$} \\
\hline Total score & $0.144^{*}$ & NS & NS & NS & $-0.156^{*}$ & NS & NS & NS \\
\hline NA score & $0.162^{*}$ & NS & NS & NS & NS & NS & NS & NS \\
\hline SI score & NS & NS & NS & NS & $-0.149^{*}$ & NS & NS & NS \\
\hline \multicolumn{9}{|c|}{ Type D personality participants $(\mathrm{N}=149)$} \\
\hline Total score & NS & NS & NS & NS & NS & NS & NS & NS \\
\hline NA score & NS & NS & NS & NS & NS & NS & NS & NS \\
\hline SI score & NS & NS & NS & NS & NS & NS & NS & NS \\
\hline
\end{tabular}

${ }^{*} \mathrm{p}<0.05$. NA: negative affectivity, SI: social inhibition, HRV: heart rate variability, SDNN: standard deviation of the normal to normal interval, RMSSD: square root of the mean squared differences of successive normal to normal intervals, TP: total power, VLF: very low frequency, LF: low frequency, HF: high frequency, LF/HF: ratio of LF to HF, NS: not significant

commonly visit community mental health centers when they feel distressed or stuck in some area of their lives and want to get some help or expert guidance from mental health professionals. Although all of the people participated in the study voluntarily and we excluded those with major cardiovascular disease, the participants in our study were more likely to have already been experiencing psychiatric distress. Therefore, it is possible that type $\mathrm{D}$ personality participants are more likely to experience psychiatric distress and so accounted for more of our study population compared to an average sample of the population.

We analyzed and compared the parameters of HRV in our participants. No significant difference was found between the type $\mathrm{D}$ group and non-type $\mathrm{D}$ group. We also examined the correlations between HRV parameters and type D total/subscale scores in both the type $\mathrm{D}$ group and the non-type $\mathrm{D}$ group after controlling for age. Age is known to have a great impact on HRV. ${ }^{22}$ HRV parameters did not show any clear correlation with type $\mathrm{D}$ personality total/subscale scores in either participant group. Although statistically significant, all of the correlation coefficients were low, which suggests that the size of the correlation is small and two variables showed have little to do with each other.

Several explanations may exist for this result, which was contrary to our expectation that HRV parameters would show correlations with type D scale scores. First, HRV recordings can be gathered with short-term, 5-minute recordings or 24hour, long-term recordings. A 5-minute resting HRV measurement could be used reliably in most clinical settings. However, there are differences in the obtained parameters between short-term and long-term recordings. Those methods cannot replace each other. ${ }^{23}$ Accordingly, the spectral analyses of 
short- and long-term HRV should always be strictly distinguished. ${ }^{24}$ Long-term HRV has its own uses; for example, ultra-low frequency components can only be assessed using a 24-hour, long-term recording. We chose a short-term analysis for clinical applicability as it was easily applied to community mental health center users. However, 5 minutes may be an inadequate amount of time to evaluate the relationship between type D personality and ANS function measured by HRV parameters. In addition, personality traits are a consequence of ongoing traits and can be seen as enduring personal characteristics of perceiving, relating to, and thinking about the environment and oneself, , $^{25}$ while $\mathrm{HRV}$ is a relatively shortterm measurement that is influenced by momentary physiologic changes. There is a possibility that long-term measurements are required to assess the psychophysiological changes derived from the specific personality trait through HRV. Therefore, further study of the correlation of long-term observation of HRV and type D personality is needed.

Second, the characteristics of participants also must be considered. We included non-clinical samples in order to focus on the relationship between HRV and type D personality without confounding factors. No significant correlations were found, which indicates that a relationship between HRV and type $\mathrm{D}$ personality parameters was not shown in non-clinical participants who do not yet have definitive cardiovascular disease. It may also be possible that a relationship between type D personality and HRV parameters might be more prominent in a clinical population that has cardiovascular disorders.

This study has some limitations. It was based on community members with no known cardiovascular diseases or psychiatric history, which may be confounding factors that could obscure study findings. The surveys were self-reports instead of structured interviews, so it is possible that past medical and psychiatric disorders were missed. The participants also were not randomly recruited from the general population, as clients of local community mental health centers voluntarily participated in the study. As a result, our results cannot be generalized to the general population. In addition, other sociodemographic variables-such as socioeconomic status-were not included in the survey because of the practical difficulties of measurement.

Type D personality can be regarded as a promising cardiovascular risk marker that has been repeatedly linked to relevant indicators of mental health, quality of life, morbidity, and mortality in cardiac patients. ${ }^{26}$ This study focused on identifying the relationship between type D and HRV and the differences in $\mathrm{HRV}$ variables between type $\mathrm{D}$ and non-type D personalities. Although short-term HRV measurements did not reveal a relationship between HRV and type D personality in a non-clinical sample without definitive cardiovascular disease, further studies using long-term HRV measurements in patients with cardiovascular disease are necessary to conclude an association between HRV and type D personality.

\section{REFERENCES}

1. Kochanek KD, XU J, Murphy SL, Minino AM, Kung HC. Deaths: final data for 2009. Natl Vital Stat Rep 2011;60:1-116.

2. Kuehn BM. Costs of cardiac care likely to increase, despite advances in prevention, care. JAMA 2013;310:2029.

3. Rozanski A, Blumenthal JA, Kaplan J. Impact of psychological factors on the pathogenesis of cardiovascular disease and implications for therapy. Circulation 1999;99:2192-2217.

4. Suls J, Bunde J. Anger, anxiety, and depression as risk factors for cardiovascular disease: the problems and implications of overlapping affective dispositions. Psychol Bull 2005;131:260-300.

5. Cloninger CR, Svrakic DM. Personality Disorders. In: Fatemi SH, Clayton PJ, Editors. The Medical Basis of Psychiatry. New York: Humana Press, 2008, p.471-483.

6. Friedman M, Rosenman RH. Association of specific overt behavior pattern with blood and cardiovascular findings; blood cholesterol level, blood clotting time, incidence of arcus senilis, and clinical coronary artery disease. J Am Med Assoc 1959;169:1286-1296.

7. Denollet J. Type D personality. A potential risk factor refined. J Psychosom Res 2000;49:255-266.

8. Denollet J. DS14: standard assessment of negative affectivity, social inhibition, and Type D personality. Psychosom Med 2005;67:89-97.

9. Denollet J, Sys SU, Brutsaert DL. Personality and mortality after myocardial infarction. Psychosom Med 1995;57:582-591.

10. Pedersen SS, Denollet J, Ong AT, Sonnenschein K, Erdman RA, Serruys PW, et al. Adverse clinical events in patients treated with sirolimus-eluting stents: the impact of Type D personality. Eur J Cardiovasc Prev Rehabil 2007;14:135-140.

11. Reich J, Schatzberg A. Personality traits and medical outcome of cardiac illness. J Psychiatr Res 2010;44:1017-1020.

12. Cohen H, Matar MA, Kaplan Z, Kotler M. Power spectral analysis of heart rate variability in psychiatry. Psychother Psychosom 1999;68:5966.

13. Akselrod S, Gordon D, Ubel FA, Shannon DC, Berger AC, Cohen RJ. Power spectrum analysis of heart rate fluctuation: a quantitative probe of beat-to-beat cardiovascular control. Science 1981;213:220-222.

14. Luecken LJ, Appelhans BM. Early parental loss and salivary cortisol in young adulthood: the moderating role of family environment. Dev Psychopathol 2006;18:295-308.

15. McCraty R, Atkinson M, Tiller WA, Rein G, Watkins AD. The effects of emotions on short-term power spectrum analysis of heart rate variability. Am J Cardiol 1995;76:1089-1093.

16. Gerritsen J, Dekker JM, TenVoorde BJ, Kostense PJ, Heine RJ, Bouter $\mathrm{LM}$, et al. Impaired autonomic function is associated with increased mortality, especially in subjects with diabetes, hypertension, or a history of cardiovascular disease: the Hoorn Study. Diabetes Care 2001;24: 1793-1798.

17. Lim HE, Lee MS, Ko YH, Park YM, Joe SH, Kim YK, et al. Assessment of the type $\mathrm{D}$ personality construct in the Korean population: a validation study of the Korean DS14. J Korean Med Sci 2011;26:116-123.

18. Malliani A, Lombardi F, Pagani M. Power spectrum analysis of heart rate variability: a tool to explore neural regulatory mechanisms. Br Heart J 1994;71:1-2.

19. O’Brien IA, O'Hare P, Corrall RJ. Heart rate variability in healthy subjects: effect of age and the derivation of normal ranges for tests of autonomic function. Br Heart J 1986;55:348-354.

20. O’Dell KR, Masters KS, Spielmans GI, Maisto SA. Does type-D personality predict outcomes among patients with cardiovascular disease? A meta-analytic review. J Psychosom Res 2011;71:199-206. 
21. Grande G, Romppel M, Barth J. Association between type D personality and prognosis in patients with cardiovascular diseases: a systematic review and meta-analysis. Ann Behav Med 2012;43:299-310.

22. Zhang J. Effect of age and sex on heart rate variability in healthy subjects. J Manipulative Physiol Ther 2007;30:374-379.

23. Task Force of the European Society of Cardiology and the North American Society of Pacing and Electrophysiology. Heart rate variability: standards of measurement, physiological interpretation and clinical use. Circulation 1996;93:1043-1065.

24. Task Force of the European Society of Cardiology and the North Ameri- can Society of Pacing and Electrophysiology. Heart rate variability. Standards of measurement, physiological interpretation, and clinical use. Eur Heart J 1996;17:354-381.

25. Hopwood CJ, Thomas KM, Markon KE, Wright AG, Krueger RF. DSM5 personality traits and DSM-IV personality disorders. J Abnorm Psychol 2012;121:424-432.

26. Denollet J, Schiffer AA Spek V. A general propensity to psychological distress affects cardiovascular outcomes: evidence from research on the type D (distressed) personality profile. Circ Cardiovasc Qual Outcomes 2010;3:546-557. 